\title{
BMJ Open Concordance of chronic conditions among the household members in Shanghai: a cross-sectional study
}

\author{
Yingyi Qin, ${ }^{1}$ Yibin Guo, ${ }^{1}$ Yuanjun Tang, ${ }^{2}$ Cheng Wu, ${ }^{1}$ Xinji Zhang, ${ }^{1}$ Qian He, ${ }^{1}$ \\ Jia $\mathrm{He}$ (1) ${ }^{1,3}$
}

To cite: Qin Y, Guo Y, Tang Y, et al. Concordance of chronic conditions among the household members in Shanghai: a crosssectional study. BMJ Open 2019;9:e031240. doi:10.1136/ bmjopen-2019-031240

- Prepublication history and additional material for this paper are available online. To view these files, please visit the journal online (http://dx.doi. org/10.1136/bmjopen-2019031240).

YQ, YG and YT contributed equally.

Received 07 May 2019

Revised 29 November 2019

Accepted 29 November 2019

Check for updates

(c) Author(s) (or their employer(s)) 2019. Re-use permitted under CC BY-NC. No commercial re-use. See rights and permissions. Published by BMJ.

${ }^{1}$ Department of Health Statistics, Second Military Medical University, Shanghai, China ${ }^{2}$ Department of Clinical Pharmacy, Shanghai General Hospital, School of Medicine, Shanghai Jiaotong University, Shanghai, China

${ }^{3}$ Tongji University School of Medicine, Shanghai, China

Correspondence to Dr Jia He; hejia63@yeah.net

\section{ABSTRACT}

Objectives Members living in the same household tend to share some similar behaviours and environment. We want to quantitatively assess the associations of chronic conditions to investigate the concordance of disease status among the household members.

Setting Shanghai, China.

Participants Our data were from the fifth Health Service Survey in Shanghai in 2013. 12002 households with 31 531 residents were selected in this survey by using a three-stage, stratified, random sampling method. Outcome measures Five highly prevalent chronic conditions, namely hypertension, diabetes, ischaemic heart disease (IHD), cerebrovascular disease (CVD) and obesity were chosen. The generalised estimating equations (GEE) model was used to estimate the associations adjusted for age, gender, education status, health insurance status, smoking and drinking. Using a subsample of adult children with parents' chronic conditions as the key risk factor and a subsample of wives with the chronic conditions of the husband as key risk factor, we reran our GEE models to explore chronic condition concordance within these relationships.

Results A total of 10198 households with 27010 adult participants were included. Using all adult household members, we found positive statistically significant associations between one's chronic conditions and the same disease status of their household members (hypertension ( $\mathrm{OR}=3.26,95 \% \mathrm{Cl} 3.02$ to 3.52); diabetes (OR=1.68, 95\% Cl 1.40 to 2.01); IHD (OR=5.31, 95\% Cl 3.56 to 7.92$)$; $\mathrm{CVD}(\mathrm{OR}=3.40,95 \% \mathrm{Cl} 1.99$ to 5.80$)$; obesity ( $\mathrm{OR}=3.41,95 \% \mathrm{Cl} 2.34$ to 4.96$))$. The results of analysing ad-child subsample and spouse subsample also showed similar associations. Moreover, the potential concordance of different chronic conditions was found between hypertension and diabetes.

Conclusions We found chronic condition concordance within households. This study provides evidence that the chronic conditions of other members of a household may be a significant risk factor for a household member's own health.

\section{BACKGROUND}

During the past three decades, China had experienced rapid social, economic and health services development. ${ }^{12}$ Life expectancy has increased, lifestyles have changed,

\section{Strengths and limitations of this study}

This is the first study in China to estimate the risk to a household member's own health, which associate with the chronic conditions of other household members.

- We perform multivariate logistic generalised estimating equations models to estimate the association between an adult's own chronic condition status and the chronic condition status of other household members within three subsamples including all adult household members, children with parents' chronic conditions as key risk factor and wives with husband's chronic conditions as key risk factor.

- The definition and diagnosis of chronic condition for each participant was based on his or her selfreported records.

- This study was conducted in Shanghai and the results might not be generalised to other regions of China.

- Based as it is on cross-sectional data, this study does not estimate the risk of a new chronic condition in a household member, nor does it provide evidence of a causal relationship.

healthcare has become more accessible and health insurance coverage has increased. Aligned with these remarkable improvements, healthcare concerns in the country have expanded from a narrow focus on infectious diseases to encompass treatment for non-communicable chronic conditions as well. ${ }^{2-4}$ Research has shown the prevalence of hypertension increased from $5.11 \%$ in 1959 to $17.65 \%$ in 2002 and the prevalence of type 2 diabetes mellitus increased from $1 \%$ in 1980 to $9.7 \%$ in $2008 .{ }^{5-7}$ In measuring disability-adjusted life-years (DALYs), noncommunicable chronic conditions have become main contributors to a country's burden of disease. As of 2015, high systolic blood pressure, high fasting plasma glucose and high body mass index (BMI), respectively ranked first, second and fourth as important risk factors related to DALY. In 1990, the 
respective ranks of these three diseases were only 3rd, 10 th and 13th. ${ }^{8}$ As a typical city in China, Shanghai represents the direction of economic and healthy development in China. Noteworthily, ageing is a significant issue in Shanghai. Moreover, this would bring about the new healthcare challenges for Chinese society and government. ${ }^{9}$

Improvement in healthcare and innovation of new effective medical treatments will definitely play an increasingly important role in dealing with chronic conditions. Additionally, detection of risk factors and identification of individuals at high-risk are the important first steps in the prevention and treatment of chronic conditions. To study the risk factors, various research has mainly focused on the personal lifestyles related to the risk of chronic conditions, for example, smoking, drinking, exercise and diet. ${ }^{10-13}$ Some genetic mechanisms are also considered as the risk factors of chronic conditions. ${ }^{14}{ }^{15}$ The household environment, which integrate the lifestyles, genetic mechanisms and environmental factors, would be an important research direction in controlling the prevalence of chronic conditions. ${ }^{16}$

Based on the fifth Health Service Survey of Shanghai, which was the extension of China's National Health Service Survey (NHSS) in 2013, the prevalence rate was $27.24 \%$ for hypertension and $7.05 \%$ for diabetes among 29269 people aged 15 years or older. ${ }^{17}$ Regarding hypertension, 6096 out of 12002 households $(50.79 \%)$ had at least one member with hypertension, and 1733 households (28.43\%) of these 6096 households had at least two members suffering from the disease. This result showed the possibility of household clustering in the prevalence of chronic conditions. We hypothesised that there were associations between chronic conditions in the participants and the same conditions in their household members, we conducted the research and analyses using data from the fifth Health Service Survey of Shanghai. In this study, we focused on five highly prevalent chronic conditions: hypertension, diabetes, ischaemic heart disease (IHD), cerebrovascular disease (CVD) and obesity. Risk factors based on chronic conditions in other family members were detected for subsamples consisting of all household members, adult children and wives, respectively.

\section{METHODS}

\section{Data source}

In this study, we used data from the fifth Health Service Survey of Shanghai in 2013. This survey was organised and conducted by the Shanghai Municipal Commission Health and Family Planning, and was the extension of NHSS. From 1993 to 2013, the NHSS has been conducted for five times (every 5 years), and is a cross-sectional survey study. ${ }^{18}$ The sampling method and quality assurance measures used in Shanghai survey were consistent with the national sampling approach and principle. ${ }^{19}$ The fifth Health Service Survey of Shanghai was conducted in all of the 17 districts in Shanghai. The survey adopted a three-stage, stratified, random sampling method. In the first stage, 100 towns/townships were randomly selected from all these 17 districts. In the second stage, 1000 villages/communities were randomly sampled from the selected towns/townships. In the third stage, about 12 000 households were randomly selected.

We conducted a face-to-face interview with each household using a structured household questionnaire developed by the National Commission Health and Family Planning of China. The questionnaire contained the general information of the households, the demographic characteristics of household members, relationships among them, their self-reported illnesses and injuries, as well as their outpatient and inpatient information.

To ensure the quality of this survey, certain assurance measures were applied during the process of data collection. Survey constitutors checked potential logical errors of the collected data. In case of any logical errors, the investigators would contact the household members and verify the relevant information. The accuracy of data information was assessed using the revisit approach. More specifically, the investigators revisited $5 \%$ of the sampled households and collected 10 key questions to check the consistency of the information recorded. The consistency rate between these two visits was nearly $99 \%$. Additionally, the Myer's Blended index was 7.39, indicating that there was non-existence of age preference in this survey. ${ }^{17} 20$ Finally, this survey collected the information of 12002 households with 31531 participants. We included the households with at least two adults aged 18 years or older.

\section{Five chronic conditions}

In this survey, we chose five chronic conditions with high prevalence rates: hypertension, diabetes, IHD, CVD and obesity. The definition of these chronic conditions was based on the corresponding questions in the questionnaire, the disease coding list of the NHSS and BMI. If a participant chose 'YES' or a specific disease code, he or she was considered to have the corresponding chronic condition.

\section{Hypertension}

Hypertension was indicated based on the question 'Have you ever been told by a doctor that you have hypertension?' in the questionnaire.

\section{Diabetes}

Diabetes was indicated based on the question 'Have you ever been told by a doctor that you have diabetes?' in the questionnaire.

\section{Ischaemic heart disease}

The disease codes for IHD included angina pectoris (061), myocardial infarction (062) and other ischaemic heart disease (063).

Cerebrovascular disease

The disease code for CVD included cerebrovascular disease (067). 
Obesity

Obesity was indicated by the WHO International BMI categories $\left(\mathrm{BMI} \geq 30 \mathrm{~kg} / \mathrm{m}^{2}\right) .^{21}$

\section{Covariates}

Sociodemographic characteristics added to our models as covariates include age (continuously specified in years), education status (illiteracy/primary, secondary or college), health insurance status (yes or no), marriage status (married, unmarried, divorced or widowed), smoking (yes or no) and drinking (yes or no). Except the analyses of spouse subsample, gender (male or female) was also included as a covariate.

\section{Statistical analyses}

This study had five primary outcomes, each of which represented the status of each of the five chronic conditions, namely hypertension, diabetes, IHD, CVD and obesity, in the participants ('No' or 'Yes'). If a participant had any of these five chronic conditions ('Yes'), his or her status of having 'Any chronic condition' is considered as 'Yes'. To make a comprehensive assessment, we settled three subsamples: all adult household members (the total sample), adult children (adult children subsample) and wives (spouse subsample). In addition, we identified chronic conditions in the other household members as the exposure (or risk factor) in three subsamples.

For the full study sample of all adult household members, we included all household members aged 18 years or older. If any other residents (excluding self) have the given chronic conditions, the exposure status of household situation for each participant is identified as 'Yes'. For the subsample of adult children, only adult children would be included in analyses, and those participants were excluded if the disease information of parents were not available. The chronic conditions status of their parents was considered as exposure. For the subsample of spouses, we included married women in the analyses. We defined the chronic conditions of wives as the outcomes and the chronic conditions of husbands as the exposure.

The generalised estimating equations (GEE) model with logit link would be used to explore the associations between chronic conditions of participants and the conditions of the others living in the same household. We considered a two-level hierarchical structure of the model (individuals within households). The model was based on individual's data without taking sampling weight into account due to the lack of relevant information. The OR and $95 \%$ CI were estimated by the GEE model to indicate the association between any chronic condition or each given chronic condition of an individual and the same condition of household member (eg, the association between the hypertensive status of a participants and that same condition in his or her other household members). In addition, the association of different chronic conditions would also be assessed (eg, the association between hypertension in a participant and diabetes in other household members).

Adjusted models and unadjusted models were both used to estimate the associations in three household subsamples. The adjusted models included age, gender, health insurance status, education status, drinking and smoking; however, gender was excluded in the subsample

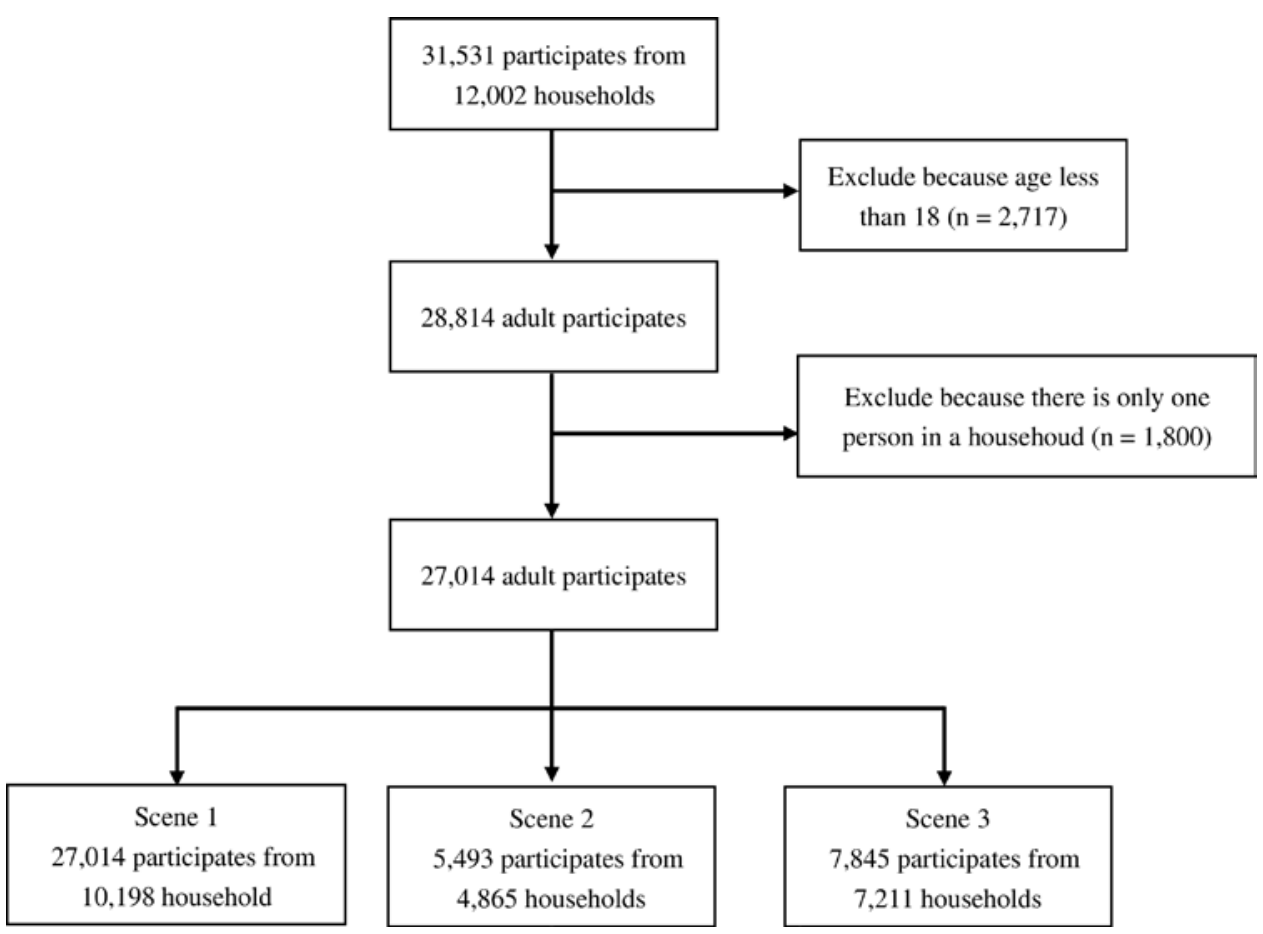

Figure 1 The flow chart of participates selection of the three subsamples analysis. There were four participates missing in education status or marriage status in scene 1. There were four participates missing in education status or marriage status in scene 2. There was one participate missing in education status or marriage status in scene 3. 
Table 1 Sociodemographic characteristics of participants

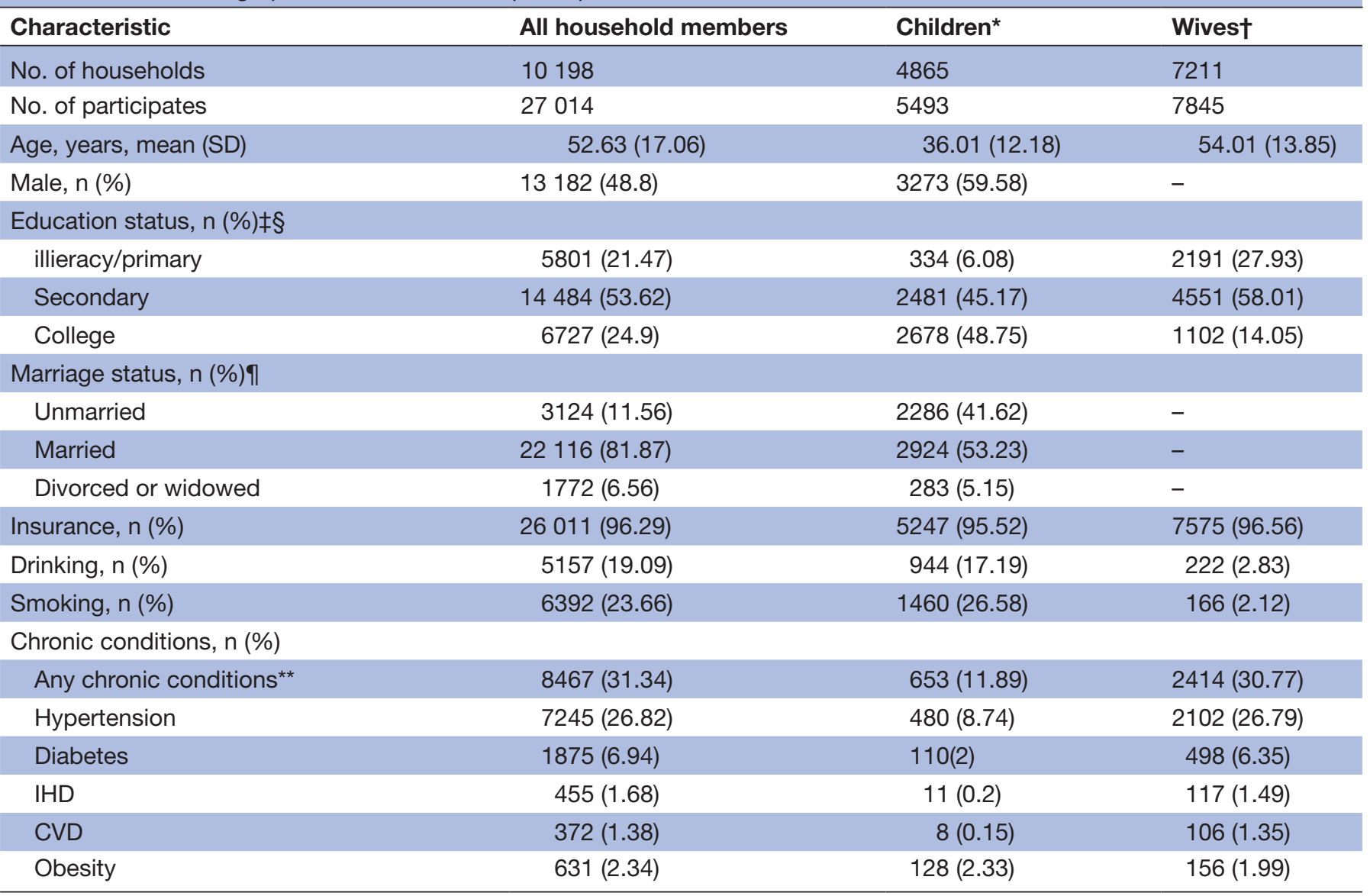

${ }^{*}$ The characteristics of children in parents-children subsample.

†The characteristics of wives in spouses subsample.

¥'lllieracy/primary' in education status means the education years were from 0 to 5 years, 'secondary' means the education years were from 6 to 12 years and 'college' means the education years of residents were higher than 12 years including undergraduate and graduate degrees.

$\S$ There were two participates missing in education status in data set.

ๆThere were two participates missing in marriage status in data set.

${ }^{* *}$ The participants had at least one chronic condition.

CVD, cerebrovascular disease; IHD, ischaemic heart disease.

of spouses. The final conclusion was based on the results of the adjusted models. The results of unadjusted models are given to provide online supplementary information. We chose the exchangeable working correlation matrix to estimate SE of coefficients in the GEE models.

The subgroup analyses were conducted for the full study sample of all adult household members according to two predefined stratification factors: sex (male or female), education (illiteracy/primary, secondary and college). We did not conduct any statistical model to deal with the missing data because of low missing data rates. Any observation with missing data would be excluded from the final analyses. All data management and statistical analyses were performed using SAS software (V.9.4). All reported $p$ values were two-sided and $p$ value $<0.05$ was regarded as statistically significant. This study was reported based on the Strengthening the Reporting of Observational studies in Epidemiology guidelines (online supplementary S1), and all analyses were conducted according to the statistical analysis plan (online supplementary S2).

\section{Patient and public involvement}

This is a cross-sectional study based on survey responses. It includes no further patient involvement.

\section{RESULTS}

A total of 10198 households (27 010 participants) with at least two adults who aged 18 years or older were included in our study from the database of the fifth Health Service Survey of Shanghai in 2013. For the subsample of adult children, there were 5489 available records, and for the subsample of spouses, there were 7844 records. The detail information was shown in flowchart (figure 1). Table 1 has shown the details of sociodemographic characteristics. The mean age of all included participants was 52.63 years, and $31.34 \%(\mathrm{n}=8467)$ had at least one chronic condition. However, the adult children participants in the subsample of adult children were young (36.01 years), and the prevalence rates of chronic conditions were much low $(11.89 \%)$. 


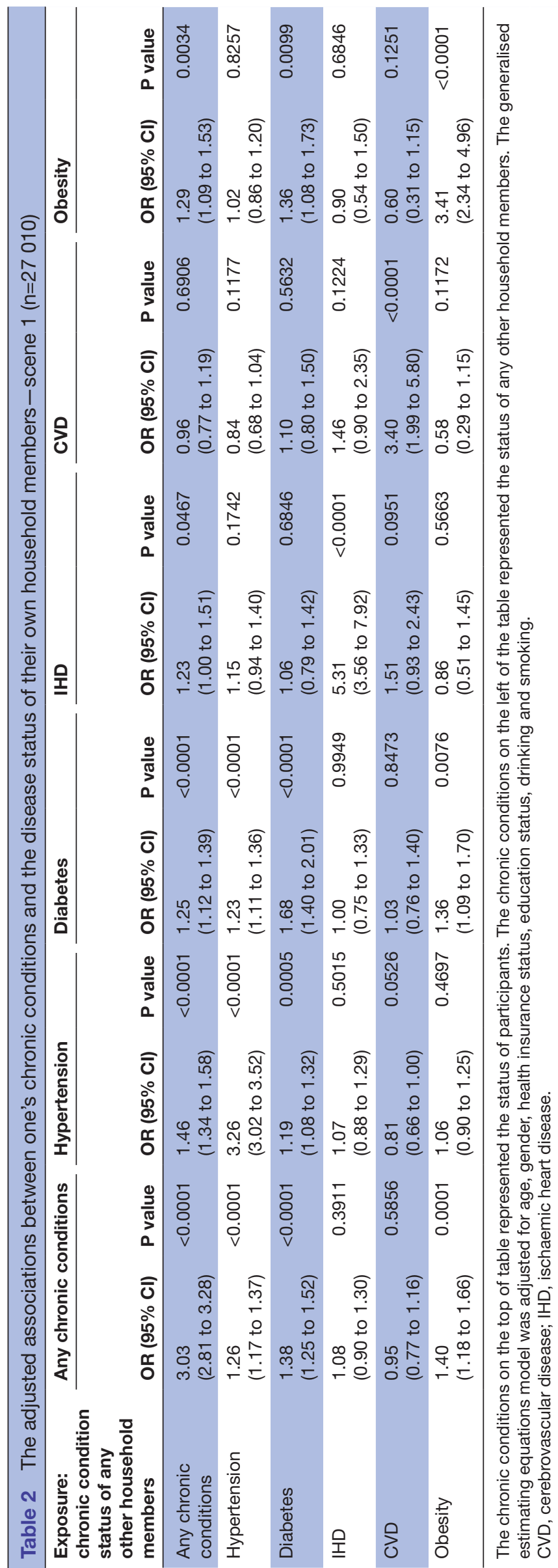

All household members

In the full study sample of all adult household members, the results indicated that the chronic conditions of participants was associated with the same conditions of others living in the same household (the diagonal of table 2): any chronic condition (OR=3.03, 95\% CI 2.81 to 3.28), hypertension ( $\mathrm{OR}=3.26,95 \%$ CI 3.02 to 3.52 ), diabetes (OR=1.68, 95\% CI 1.40 to 2.01), IHD (OR $=5.31,95 \%$ CI 3.56 to 7.92$)$, CVD (OR=3.40, 95\% CI 1.99 to 5.80$)$ and obesity (OR=3.41, 95\% CI 2.34 to 4.96$)$. The results also revealed that there were significantly positive associations for some different chronic conditions (diabetes and hypertension, diabetes and obesity). The OR was 1.19 (95\% CI 1.08 to 1.32 ) for diabetes to hypertension and 1.23 (95\% CI 1.11 to 1.36 ) for hypertension to diabetes. The OR was 1.36 (95\% CI 1.08 to 1.73 ) for diabetes to obesity and 1.36 (95\% CI 1.09 to 1.70 ) for obesity to diabetes. More details were presented in table 2, and the results of unadjusted GEE models were listed in online supplementary table S1.

In subgroup analysis, we found the similar associations for the sex subgroups and education level subgroups (figures 2 and 3 and online supplementary table S2). However, no significant association was not found in the analyses of diabetes and IHD in the college education subgroup.

\section{Adult children subsample}

We did not conduct the analyses of IHD and CVD in this household subsample because of low prevalence rates $(0.2 \%$ for IHD and $0.15 \%$ for CVD). Table 3 listed the results of adjusted GEE models for adult children. The positive associations between chronic conditions of adult children and the same conditions of their parents were observed: any chronic condition $(\mathrm{OR}=5.13,95 \%$ CI 4.20 to 6.28$)$, hypertension ( $\mathrm{OR}=6.29,95 \%$ CI 4.92 to 8.05$)$, diabetes (OR=11.56, 95\% CI 7.88 to 16.98$)$ and obesity (OR=13.85, 95\% CI 8.54 to 22.46). For different chronic conditions, the children with diabetes and obesity were associated with parents with hypertension (OR was 2.40 for diabetes and OR was 1.69 for obesity). And the results also indicated that hypertension status of children was associated with the diabetes status of their parents $(\mathrm{OR}=1.61)$. The results of unadjusted GEE models for the subsample of adult children were listed in online supplementary table S3.

\section{Spouses subsample}

The results of the spouses subsample are shown in table 4 . Similar to the other two subsamples above, positive statistically significant associations were also observed between chronic conditions in husbands and the same conditions in their spouses: any chronic condition $(\mathrm{OR}=1.58,95 \%$ CI 1.41 to 1.77 ), hypertension ( $\mathrm{OR}=1.63,95 \%$ CI 1.45 to 1.84 ), diabetes (OR=1.57, 95\% CI 1.21 to 2.03$)$, IHD $(\mathrm{OR}=6.58,95 \%$ CI 3.78 to 11.43$), \mathrm{CVD}(\mathrm{OR}=3.62,95 \% \mathrm{CI}$ 1.86 to 7.07 ) and obesity (OR=5.09, 95\% CI 2.92 to 8.86 ). The wives with diabetes and IHD were associated with the hypertension status of their husbands (OR was 1.46 for diabetes and $\mathrm{OR}$ was 1.60 for IHD). And the association 


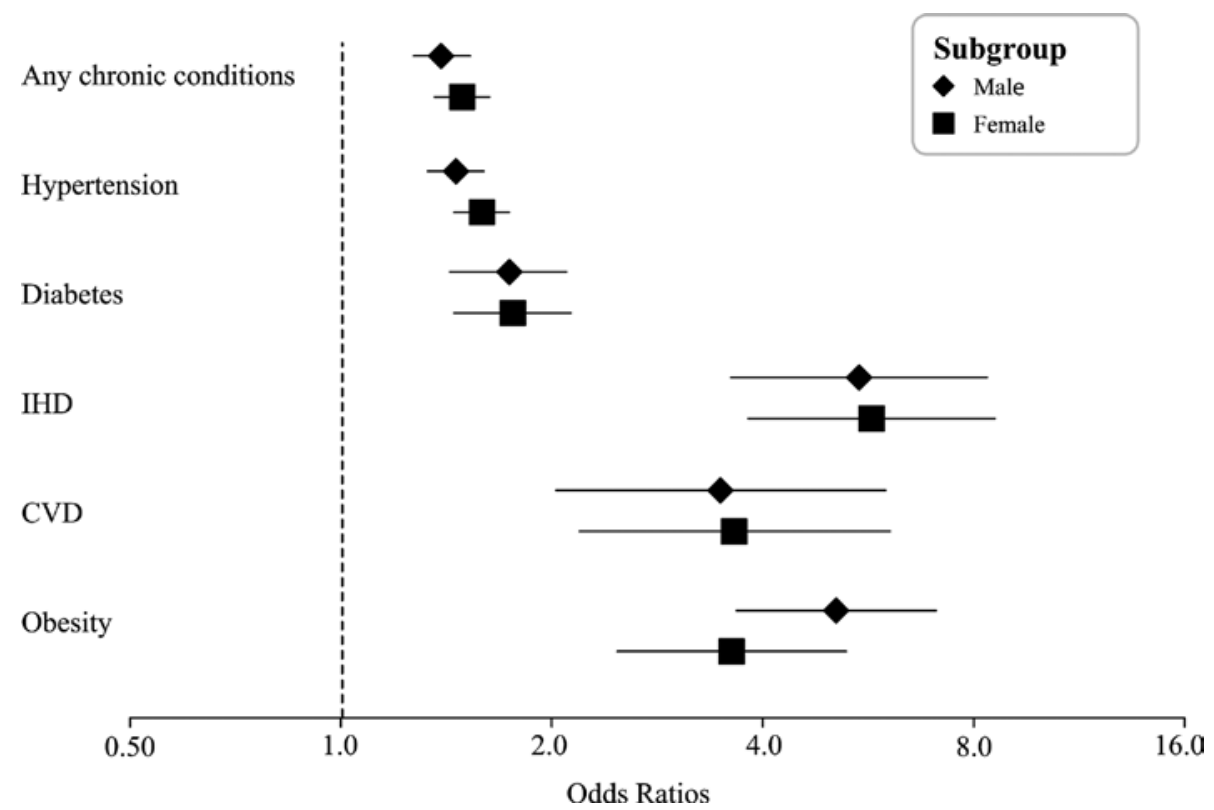

Figure 2 The forest plot of five chronic conditions subgroup analysis categorised by gender. The plot showed the adjusted ORs and $95 \% \mathrm{Cl}$ of the association between each given chronic condition of individual with the same condition of household member. CVD, cerebrovascular disease; IHD, ischaemic heart disease.

between the hypertension status of wives and the diabetes status of husbands was also indicated $(\mathrm{OR}=1.29)$. The information of unadjusted GEE models could be available in online supplementary table S4.

\section{DISCUSSION}

In China, Shanghai has the heaviest burden of noncommunicable diseases because of its largest population and ageing population. ${ }^{22}$ We conducted in-depth analyses

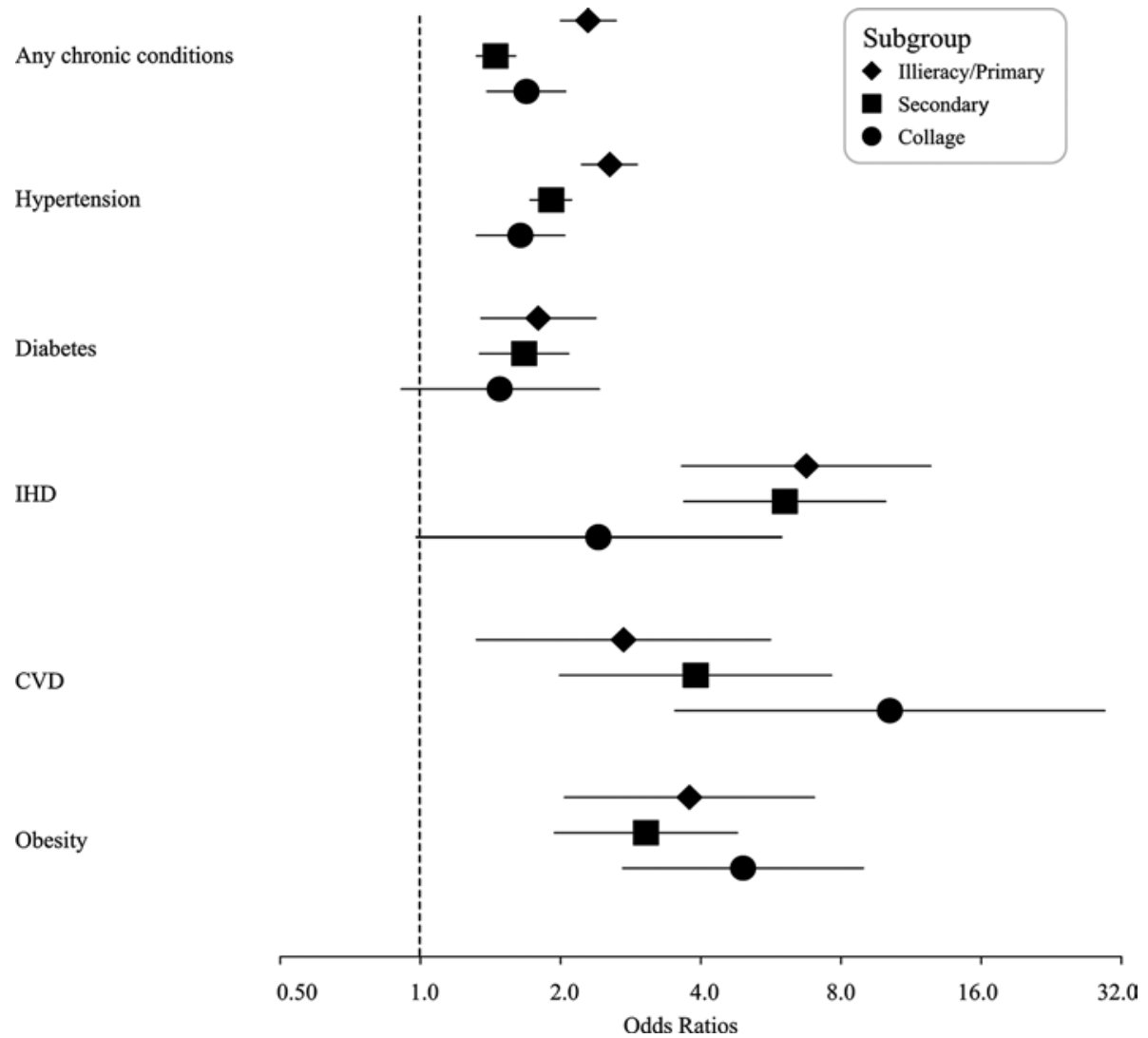

Figure 3 The forest plot of five chronic conditions subgroup analysis categorised by education status. The plot showed the adjusted $\mathrm{ORs}$ and $95 \% \mathrm{Cl}$ of the association between each given chronic condition of individual with the same condition of household member. CVD, cerebrovascular disease; IHD, ischaemic heart disease. 


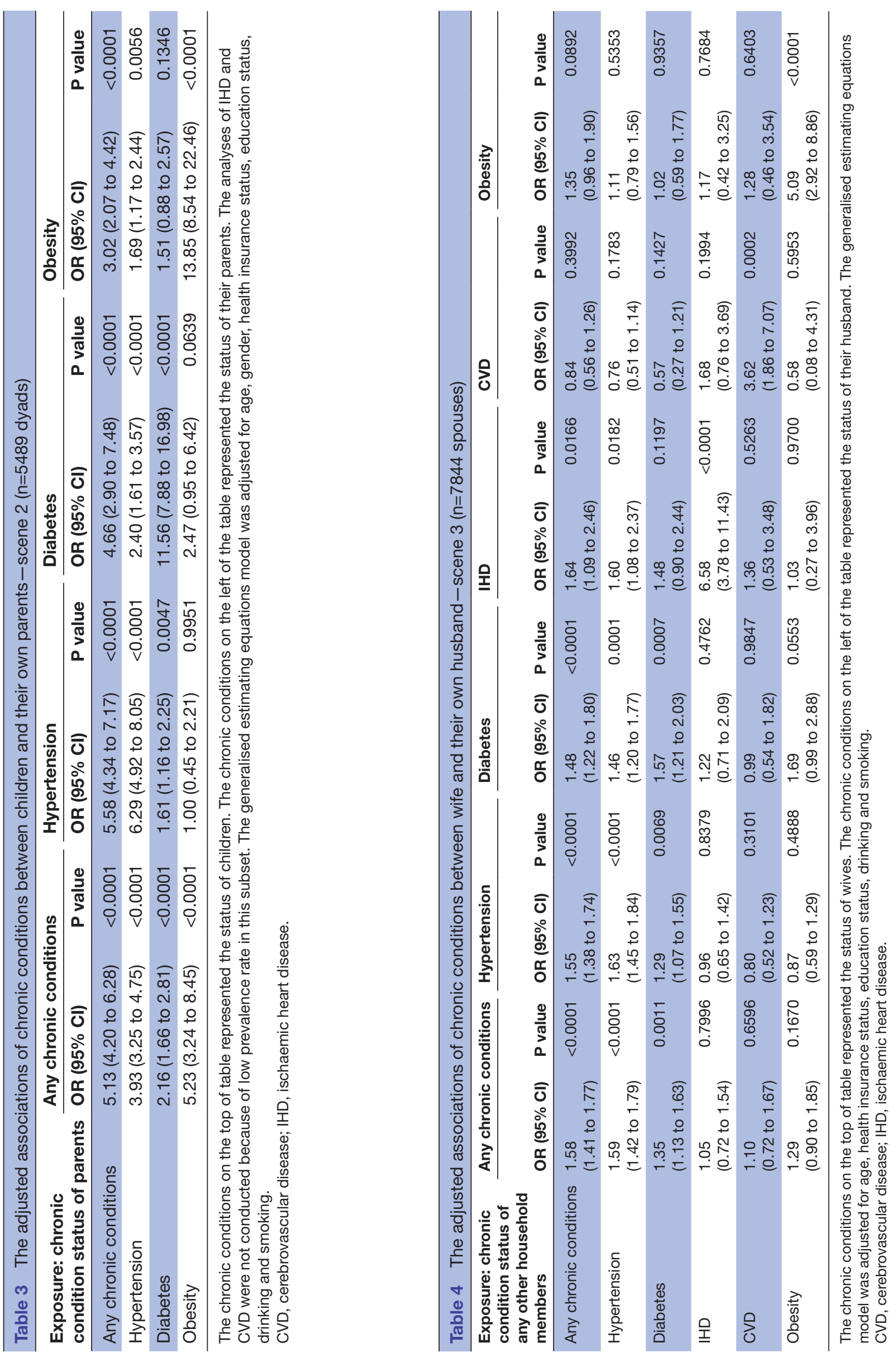


of the associations among five preselected chronic conditions status within the household members in Shanghai, China. Using the data of the fifth Health Service Survey, we found that the participants living with their household members with chronic conditions were associated with $46 \%$ higher OR of having one or more chronic conditions. For each of five chronic conditions, the above associations were observed in each same chronic condition. We also found the similar associations in children subsample and spouses subsample. These results were consistent with some other research results. ${ }^{16} 23-25$ Additionally, the results suggested potential associations between the different chronic conditions, for example, hypertension and diabetes.

We chose five chronic conditions (hypertension, diabetes, IHD, CVD and obesity) as the target diseases of our study because of the highest prevalence rates in this health service survey. Based on the current knowledge, the incidence of the chronic conditions is the result of the combination of multiple factors. The members of the same household would live in the same environment, and might have the similar behaviours. In addition, some of them would have genetic associations (eg, parents and children, or brothers and sisters). Some research reported the association between the family health history and risk of disease. ${ }^{26-28}$ However, if a member of the household was diagnosed with a certain type of chronic condition, the likelihood of the other family members having their diagnostic tests performed was higher. This phenomenon might cause reporting bias and lead to the association of chronic conditions among household members. Our study pointed out the positive associations between chronic conditions in adults and the same conditions in their household members. According to the results of all household members, we found that the ORs for IHD and CVD were high, and the 95\% CI of the corresponding ORs was wide. An explanation might be that the prevalence rates of IHD and CVD were only $1.68 \%$ and $1.38 \%$, respectively. Moreover, low prevalence rates might reduce the accuracy of the statistical model estimation. However, the results of both the adjusted GEE model and the unadjusted one showed the positive associations for these two chronic conditions.

In order to further explore the effect of a common genetic factor or a common living environment, we analysed two subsamples, a subsample of adult children to examine the two factors together, and a subsample of spouses to examine living environment factors in the absence of a common genetic link. Similar to results using all adult household members, positive associations were found in these two subsamples. These findings were consistent with some previous studies. ${ }^{23-2529} 30$ The results of analyses in these two special subsamples showed the effects of genetic factors and those of a common living environment without genetic ties on the associations of interest. Moreover, the adjusted ORs in analyses about children subsample were much higher (eg, OR=6.12 for hypertension association). However, we could make a conclusion that the effect of genetic factors was much more important. This might be because certain chronic conditions are age-related diseases, and the prevalence rates of adult children in this health service survey were low. For example, the prevalence of hypertension among adult children was only $8.86 \%$ (the mean of age was 39.16 years old), and prevalence rate for those whose parents had no hypertension was much lower (only 3.34\%, the mean of age was 34.36 years old). Based on this consideration, we included the age covariate into the adjusted model. Therefore, these results might reflect the associations between chronic conditions in children and those in their parents despite high ORs. In order to show the effects of covariates more clearly, we listed the results of full models for three subsamples in online supplementary table S5. We found that the effects of most covariates were consistent across three subsamples.

In addition to explore the association of the same chronic condition between participants and their household members, the results of different chronic conditions indicated that there was positive association between hypertension and diabetes. This association was seen in all three subsamples. The mechanisms and pathways could not be concluded in our study, but certain previous studies might provide some hypotheses. The hypertension and diabetes are the syndromes of metabolic syndrome (MetS). Some risk factors for MetS, such as lifestyle, diet, family disease history, environment, might be the cofactors for the prevalence of hypertension and diabetes. ${ }^{31-33}$ However, the exact mechanisms should need further investigation.

Although the aim of our study is not to investigate certain specific risk factors for the prevalence of chronic conditions, the quantitative assessment showed the concordance of chronic conditions within households. We suggested that individuals whose household members had certain chronic conditions should pay more attention to their health status. Health education about chronic diseases and breaking some potential unhealthy behaviours might help reduce the prevalence rates of chronic conditions among those individuals. Moreover, regular health examination (eg, measurement of blood pressure and weight) would be helpful for residents to monitor their own health status. Shanghai is the biggest and most developed city in China, and lends the development trend. Some researchers indicated that Shanghai Chronic Disease Self-Management Programme, the most widely accepted self-management patient education programmer worldwide, could improve participants' health behaviour, self-efficacy and health status. ${ }^{22}$ Maybe this programme could be modified and acceptable to the healthy residents whose household members have chronic conditions. Moreover, the chronic condition concordance within households should also be given special care in other regions of China, and the relevant solutions should be worked out advanced. These would be beneficial for controlling the prevalence rate of chronic conditions and reducing the disease burden for the country and among residents.

Our study had several potential limitations. First, the definition and diagnosis of chronic condition for each participant was based on his or her self-reported records. 
This might lead to the potential risk of under-reporting and misreporting. However, this survey was part of the NHSS and every investigator had undergone rigorous and formal training. Previous research has shown the agreement between biomedical and self-reported measurements of certain chronic conditions in a Chinese national community sample. ${ }^{34}$ Therefore, the records of these five chronic conditions would be reliable. Second, this study was conducted in Shanghai and the results could not represent the situation in other regions of China. Nevertheless, it could draw the attention of the public and health management departments to this problem. Third, our study could neither estimate the risk of new chronic conditions among household members nor find out a certain significant risk factor. We only pointed out the phenomenon that there were associations for the prevalence of chronic conditions at the household level. The mechanism and specific causes of cohabitation effects on prevalence of chronic conditions could not be presented by our study. These results suggested that we better pay attention to the health status of the health residents in the households with members with chronic conditions. Fourth, this study is a cross-sectional survey study, and several potential bias, such as recall bias, confounding bias and reporting bias, might not be completely avoided. The exclusion of households with only one family member might also cause some selection bias. To deal with the confounding bias, we conducted the analyses using the adjusted GEE models with some sociodemographic covariates.

\section{CONCLUSIONS}

In conclusion, our study indicated that one's chronic condition status was associated with the status of their household members in the Shanghai, China. The results of adult children and spouses subsamples were consistent with those of all household members. Additionally, analyses about different chronic conditions suggested possible positive associations between hypertension and diabetes. The mechanisms about these associations should be investigated by further research. However, the evidence about the association of chronic conditions for the same household members suggests that people should pay more attention to their health status, especially those whose household members have chronic conditions. That might reduce the prevalence and increase the early detection rate about some chronic conditions.

Acknowledgements We appreciate the Shanghai Municipal Commission Health and Family Planning for their assistance in designing this study. We also thank the participates and data managers who gave their time and effort to participate in the fifth Health Service Survey of Shanghai.

Contributors $Y Q, Y G$ and $J H$ designed this study. $Y Q, Y G$ and $C W$ analysed the data. $Q H$ and $X Z$ checked the statistical analysis. $Y G$ and $Y Q$ wrote the manuscript. $Y T, Y Q$, $\mathrm{YG}$ and $\mathrm{JH}$ revised this manuscript.

Funding This study was conducted under a grant from the Fourth Round of Shanghai Three-year Action Plan on Public Health Discipline and Talent Program: Evidence-based Public Health and Health Economics (No. 15GWZK0901), Shanghai
Sailing Program (No. 18YF1429500), National Thirteenth Five Year Plan Major Special Project (2017ZX09304016) and National Science and Technology Major Project (Grant No. 2017ZX09304030).

Competing interests None declared.

Patient consent for publication Obtained.

Ethics approval This cross-sectional questionnaire survey was organized by the Shanghai Municipal Commission Health and Family Planning. As we knew, this survey didn't contain any treatment, blood drawing or others intervention which might influence the health of participators. Informed consent was obtained by surveyors prior to data collection and personal information was with strict conservation during our analysis process.

Provenance and peer review Not commissioned; externally peer reviewed.

Data availability statement Data are available upon reasonable request.

Open access This is an open access article distributed in accordance with the Creative Commons Attribution Non Commercial (CC BY-NC 4.0) license, which permits others to distribute, remix, adapt, build upon this work non-commercially, and license their derivative works on different terms, provided the original work is properly cited, appropriate credit is given, any changes made indicated, and the use is non-commercial. See: http://creativecommons.org/licenses/by-nc/4.0/.

ORCID iD

Jia He http://orcid.org/0000-0002-2338-9501

\section{REFERENCES}

1 Meng Q, Xu L, Zhang Y, et al. Trends in access to health services and financial protection in China between 2003 and 2011: a crosssectional study. The Lancet 2012;379:805-14.

2 Zhou M, Wang $\mathrm{H}$, Zhu J, et al. Cause-Specific mortality for 240 causes in China during 1990-2013: a systematic subnational analysis for the global burden of disease study 2013. The Lancet 2016;387:251-72.

3 Milner J, Wilkinson P. Trends in cause-specific mortality in Chinese provinces. The Lancet 2016;387:204-5.

4 GBD 2015 Healthcare Access and Quality Collaborators. Electronic address: cjlm@uw.edu, GBD 2015 Healthcare Access and Quality Collaborators. Healthcare access and quality index based on mortality from causes amenable to personal health care in 195 countries and territories, 1990-2015: a novel analysis from the global burden of disease study 2015. Lancet 2017;390:231-66.

5 Ruan Y, Mo M, Joss-Moore L, et al. Increased waist circumference and prevalence of type 2 diabetes and hypertension in Chinese adults: two population-based cross-sectional surveys in Shanghai, China. BMJ Open 2013;3:e003408.

6 Wu Y, Huxley R, Li L, et al. Prevalence, awareness, treatment, and control of hypertension in China: data from the China national nutrition and health survey 2002. Circulation 2008;118:2679-86.

7 Yang W, Lu J, Weng J, et al. Prevalence of diabetes among men and women in China. N Engl J Med 2010;362:1090-101.

8 Cohen AJ, Brauer M, Burnett R, et al. Estimates and 25-year trends of the global burden of disease attributable to ambient air pollution: an analysis of data from the global burden of diseases study 2015 . The Lancet 2017;389:1907-18.

9 Fang EF, Scheibye-Knudsen M, Jahn HJ, et al. A research agenda for aging in China in the 21st century. Ageing Res Rev 2015;24:197-205.

10 Orozco LJ, Buchleitner AM, Gimenez-Perez G, et al. Exercise or exercise and diet for preventing type 2 diabetes mellitus. Cochrane Database Syst Rev 2008;22.

11 Yu R, Yan LL, Wang H, et al. Effectiveness of a community-based individualized lifestyle intervention among older adults with diabetes and hypertension, Tianjin, China, 2008-2009. Prev Chronic Dis 2014;11:E84.

12 Pan A, Wang Y, Talaei M, et al. Relation of active, passive, and quitting smoking with incident type 2 diabetes: a systematic review and meta-analysis. Lancet Diabetes Endocrinol 2015;3:958-67.

13 Knott C, Bell S, Britton A. Alcohol consumption and the risk of type 2 diabetes: a systematic review and dose-response meta-analysis of more than 1.9 million individuals from 38 observational studies. Diabetes Care 2015;38:1804-12.

14 Mahajan A, Go MJ, Zhang W, Replication DIG, Meta-analysis C, et al. Genome-Wide trans-ancestry meta-analysis provides insight into the genetic architecture of type 2 diabetes susceptibility. Nat Genet 2014;46:234-44. 
15 Zhang C, Wang L, Liao Q, et al. Genetic associations with hypertension: meta-analyses of six candidate genetic variants. Genet Test Mol Biomarkers 2013;17:736-42.

16 Patel SA, Dhillon PK, Kondal D, et al. Chronic disease concordance within Indian households: a cross-sectional study. PLoS Med 2017; 14:e1002395.

17 Zhang $\mathrm{X}$, Zhang $\mathrm{Y}$, Xiao X, et al. The relation between health insurance and management of hypertension in Shanghai, China: a cross-sectional study. BMC Public Health 2016;16:959.

18 Yuefeng L, Keqin R, Xiaowei R. Use of and factors associated with self-treatment in China. BMC Public Health 2012;12:995.

$19 \mathrm{Xu}$ Y, Gao J, Zhou Z, et al. Measurement and explanation of socioeconomic inequality in catastrophic health care expenditure: evidence from the rural areas of Shaanxi Province. BMC Health Serv Res 2015;15:256.

20 Bello Y. Error Detection in Outpatients' Age Data Using Demographic Techniques. Int J Pure Appl Sci Technol 2012;10:27-36.

21 WHO. Obesity: preventing and managing the global epidemicReport of a who consultation (who technical report series 894); 2000: 1-253.

22 Fu D, Fu H, McGowan P, et al. Implementation and quantitative evaluation of chronic disease self-management programme in Shanghai, China: randomized controlled trial. Bull World Health Organ 2003;81:174-82.

23 Lee MH, Kim HC, Thomas GN, et al. Familial concordance of metabolic syndrome in Korean population--Korean National Health and Nutrition Examination Survey 2005. Diabetes Res Clin Pract 2011;93:430-6.

24 Okuda T, Miyazaki T, Sakuragi S, et al. Significant but weak spousal concordance of metabolic syndrome components in Japanese couples. Environ Health Prev Med 2014;19:108-16.
25 Sun J, Lu J, Wang W, et al. Prevalence of diabetes and cardiometabolic disorders in spouses of diabetic individuals. Am J Epidemiol 2016;184:400-9.

26 Baptiste-Roberts K, Gary TL, Beckles GLA, et al. Family history of diabetes, awareness of risk factors, and health behaviors among African Americans. Am J Public Health 2007;97:907-12.

27 Carroll JC, Campbell-Scherer D, Permaul JA, et al. Assessing family history of chronic disease in primary care: prevalence, documentation, and appropriate screening. Can Fam Physician 2017;63:e58-67.

28 Welch BM, Dere W, Schiffman JD. Family health history: the case for better tools. JAMA 2015;313:1711-2.

29 Park HS, Park JY, Cho S-I. Familial aggregation of the metabolic syndrome in Korean families with adolescents. Atherosclerosis 2006;186:215-21.

30 Di Castelnuovo A, Quacquaruccio G, Donati MB, et al. Spousal concordance for major coronary risk factors: a systematic review and meta-analysis. Am J Epidemiol 2009;169:1-8.

31 Wilson PWF, D'Agostino RB, Parise $\mathrm{H}$, et al. Metabolic syndrome as a precursor of cardiovascular disease and type 2 diabetes mellitus. Circulation 2005;112:3066-72.

32 Buscemi S, Sprini D, Grosso G, et al. Impact of lifestyle on metabolic syndrome in apparently healthy people. Eat Weight Disord 2014;19:225-32.

33 Kim M-J, Lim N-K, Choi S-J, et al. Hypertension is an independent risk factor for type 2 diabetes: the Korean genome and epidemiology study. Hypertension Research 2015;38:783-9.

34 Ning M, Zhang Q, Yang M. Comparison of self-reported and biomedical data on hypertension and diabetes: findings from the China health and retirement longitudinal study (CHARLS). BMJ Open 2016;6:e009836. 